\title{
VARIACIÓN DEL RIESGO DE INUNDACIONES EN EL RÍO ZADORRA (PAÍS VASCO) COMO CONSECUENCIA DE SU REGULACIÓN
}

\author{
Askoa Ibisate González de Matauco \\ Dpto. de Geografía, Prehistoria y Arqueología \\ Universidad del País Vasco
}

\section{RESUMEN}

La modificación del funcionamiento hidrológico del río Zadorra a raíz de la construcción y puesta en explotación de los embalses del sistema Zadorra, ha supuesto una variación en la localización espacial, intensidad y peligrosidad de los eventos de crecidas, además de una modificación de la vulnerabilidad de los asentamientos y actividades localizadas en la llanura de inundación, y por tanto en el riesgo que entrañan estos procesos.

Palabras clave: Inundaciones, riesgo, peligrosidad, vulnerabilidad, embalse, río Zadorra.

\section{ABSTRACT}

Since Zadorra reservoirs came into operation, Zadorra river's hydrology has notoriously changed. This has been also seen in the spatial location, intensity and danger of floods, and furthermore in the modification of the vulnerability of settlements and activities placed on the floodplain, and therefore the risk of these processes.

Key words: Floods, risk, danger, vulnerability, reservoir, Zadorra river.

\section{Introducción}

El riesgo de inundaciones viene dado por la conjunción de la peligrosidad de las crecidas por el incremento de la altura del agua y la vulnerabilidad del territorio sobre el cual se produce la expansión de las aguas. Hasta hace unas décadas la población ha respetado esos lugares inundables, protegiéndose así de su amenaza. Sin embargo la presión sobre unos determinados espacios, el desarrollo tecnológico y la necesidad de situarse cerca de los ríos, bien para la obtención de energía, como para la utilización del agua en procesos productivos 
o como desagüe, generó una invasión de terrenos pertenecientes a los cursos fluviales. En el caso del río Zadorra se añade otro factor que ha incrementado el riesgo, la puesta en explotación de los embalses del sistema Zadorra. Por un lado han aumentado la peligrosidad de las crecidas aguas abajo de los embalses debido a los problemas de gestión que presenta y al nuevo funcionamiento hidrológico del río, y por otro lado su construcción ha generado una falsa sensación de seguridad que ha propiciado la invasión de terrenos inundables e incluso del propio cauce. Para paliar estos efectos se siguen planteando medidas que reduzcan la peligrosidad, es decir, medidas estructurales, las cuales ante fuertes crecidas no reducen los daños sino todo lo contrario (WHITE et al, 1958), debido a los mayores efectos que se producen al rebasarse los umbrales para las que fueron diseñadas, y especialmente porque también contribuyen a esa falsa sensación de seguridad, antes comentada, propiciando la invasión de espacios que antes se respetaban por el temor a las crecidas.

El estudio del riesgo de inundaciones ha sido abordado desde diversos puntos de vista y por diferentes autores (MATEU, 1990, 1992; SAURÍ y RIBAS, 1994) centrándose en algunos casos en eventos concretos desde aspectos geomorfológicos y de procesos (ROSSELLÓ, 1983, 1989; GARCÍA-RUIZ et al, 1996), climáticos (CAPEL MOLINA, 1983; GARCÍA-RUIZ et al, 1983; OLCINA, 1994) o hidrológicos (HERAS, 1970; FERRER, 2000) y de zonificación (FERRER, 2000; BESCÓS y CAMARASA, 1998, 2000; MASSON, 1996; FRANCÉS, 1997; GILARD, 1997,1998; LAÍN, 1999), incluso un tratamiento del riesgo desde un punto de vista más social CALVO $(1984,1986,1997,2001)$. Asimismo destacan importantes obras recopilatorias como las de BAKER et al (1988), BEVEN et al (1989), GIL OLCINA y MORALES GIL (1989), KUNDZEWICZ et al (1993) y CAMARASA y MATEU (2000). O monográficos sobre riesgos naturales donde se analizan también las crecidas y el riesgo de inundaciones como los de AYALA et al (1987) y AYALA y OLCINA (2002).

\section{Funcionamiento hidrológico del río Zadorra}

El río Zadorra, situado en la zona de cabecera de la cuenca del Ebro y en su margen izquierda (Figura 1), cuenta con una cuenca de drenaje de $1.361,28 \mathrm{~km}^{2}$, una longitud de 78 $\mathrm{kms}$ y un caudal medio de $14,1 \mathrm{~m}^{3} / \mathrm{s}$ (IBISATE, 2004). En el centro de la cuenca se localizan los embalses del sistema Zadorra, éstos van a condicionar el funcionamiento del río aguas abajo de los mismos desde el inicio de su explotación en 1956 (Figura 2). El sistema cuenta con cuatro embalses interconectados entre sí: Ullibarri-Ganboa, Urrunaga, Albina y Undurraga, que presentan una complicada gestión, dado que son múltiples los usos para los que se destinan, generación hidroeléctrica, abastecimiento urbano-industrial y laminación de avenidas, y que regulan $416 \mathrm{~km}^{2}$ de cuenca.

Los embalses de Ullibarri y Urrunaga son los de mayor capacidad, con 147 y $72 \mathrm{Hm}^{3}$ respectivamente y se sitúan junto con el de Albina en la vertiente mediterránea. El primero vierte directamente sus aguas al río Zadorra, $6 \mathrm{kms}$ aguas arriba de la ciudad de VitoriaGasteiz, el segundo al río Santa Engracia, que confluye en el río Zadorra $3 \mathrm{kms}$ aguas arriba de la misma ciudad, y el tercero al río Albina, aguas que son recogidas posteriormente por el embalse de Urrunaga. El cuarto embalse, Undurraga se sitúa en la vertiente cantábrica, más concretamente en el valle de Arratia, a través del cual se realiza el salto hidroeléctrico para la obtención de energía y mediante el que también se hace el trasvase de aguas del Zadorra al Arratia para el abastecimiento del Gran Bilbao. A la dificultad de gestionar unos embalses con diversos usos, se une la maraña de concesiones, propietarios y derechos, e intereses contrapuestos que dificultan más si cabe la toma de decisiones para afrontar el riesgo. 
Variación del riesgo de inundación en el río Zadorra (País Vasco) como consecuencia de su regulación

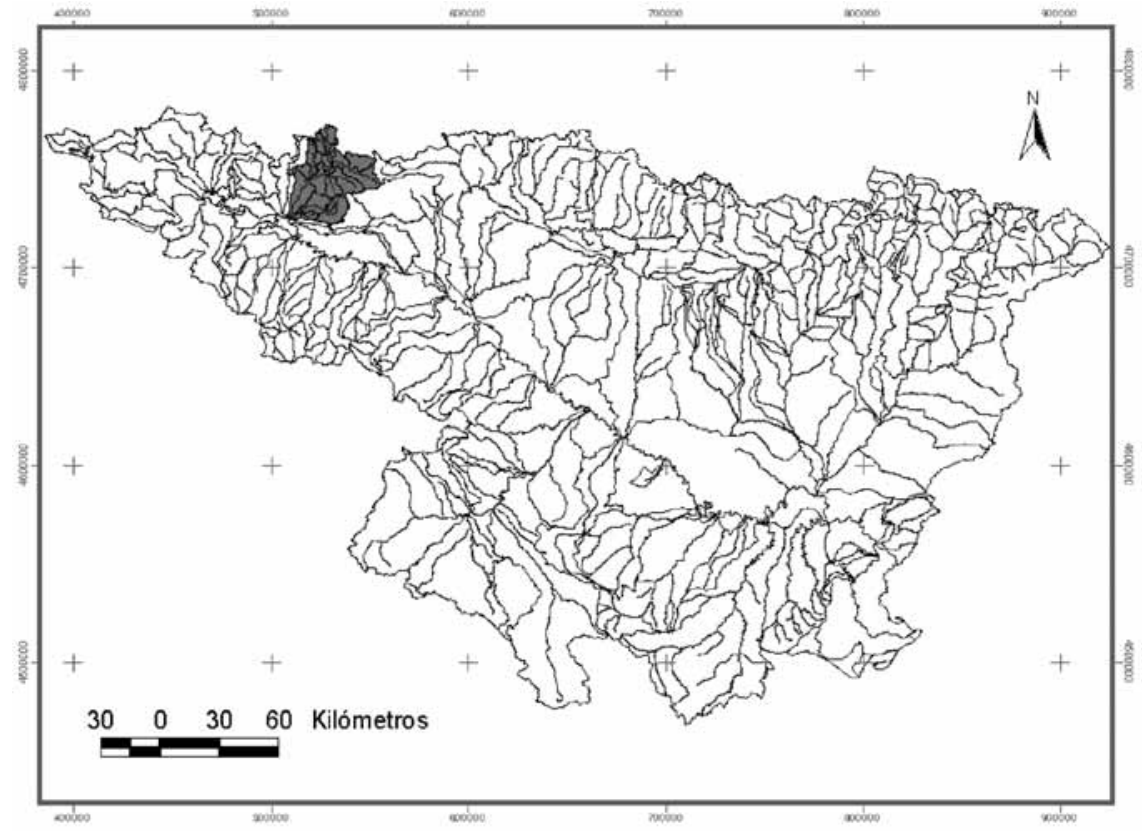

FIgURA 1. La cuenca del río Zadorra en la cuenca del río Ebro. (Fuente: Confederación Hidrográfica del Ebro. Elaboración propia).

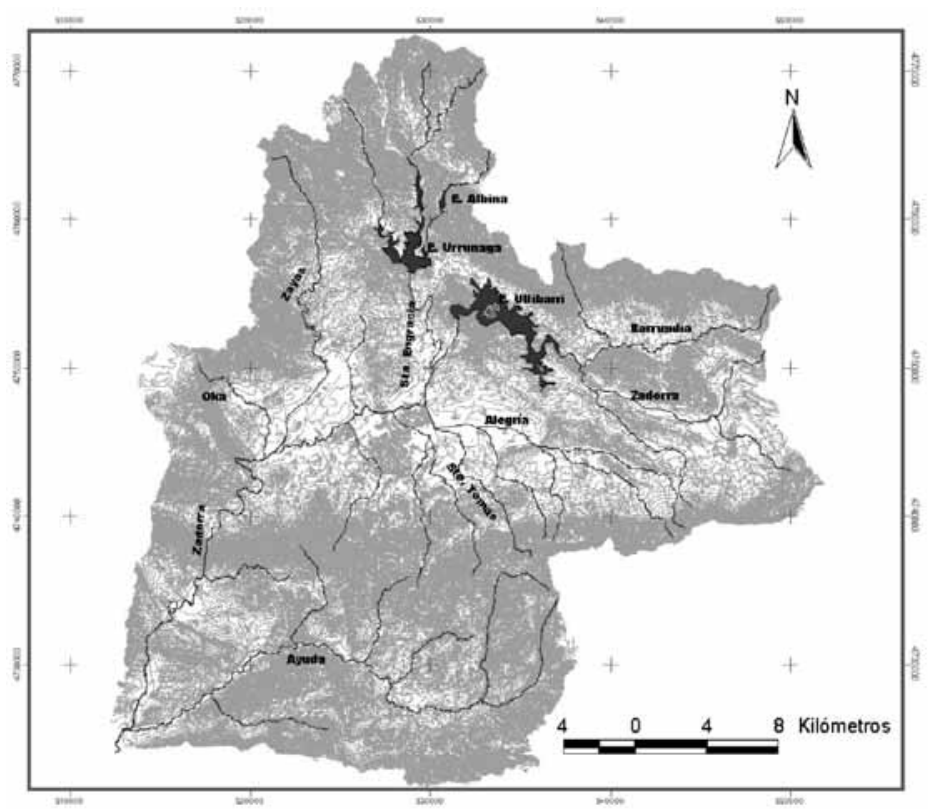

FiguRa 2. La cuenca del río Zadorra. (Fuente: Gobierno Vasco. Elaboración propia). 
La Figura 3 muestra la modificación que este sistema de embalses ha producido en el funcionamiento hidrológico del río, y especialmente en el de sus eventos extremos, crecidas y estiajes. Por un lado se ha producido una drástica reducción de los caudales, especialmente durante el periodo de aguas altas, pasando durante el mes de febrero de $50,8 \mathrm{~m}^{3} / \mathrm{s} \mathrm{de}$ caudal medio a $20,5 \mathrm{~m}^{3} / \mathrm{s}$, de manera que el trasvase hacia la vertiente cantábrica ha supuesto un descabezamiento del río Zadorra (MARTÍNEZ, 2003). Sin embargo, la propia regulación y el mantenimiento de los denominados caudales ecológicos ha producido un incremento de los caudales medios durante el periodo de estiaje, lo que ha generado que por el río circule más agua de la que naturalmente llevaría, pasando en agosto de $2,4 \mathrm{~m}^{3} / \mathrm{s}$ a 3,0 $\mathrm{m}^{3} / \mathrm{s}$. De hecho en algunos puntos, el río se quedaba en determinados días seco, circunstancia que ahora no sucede.

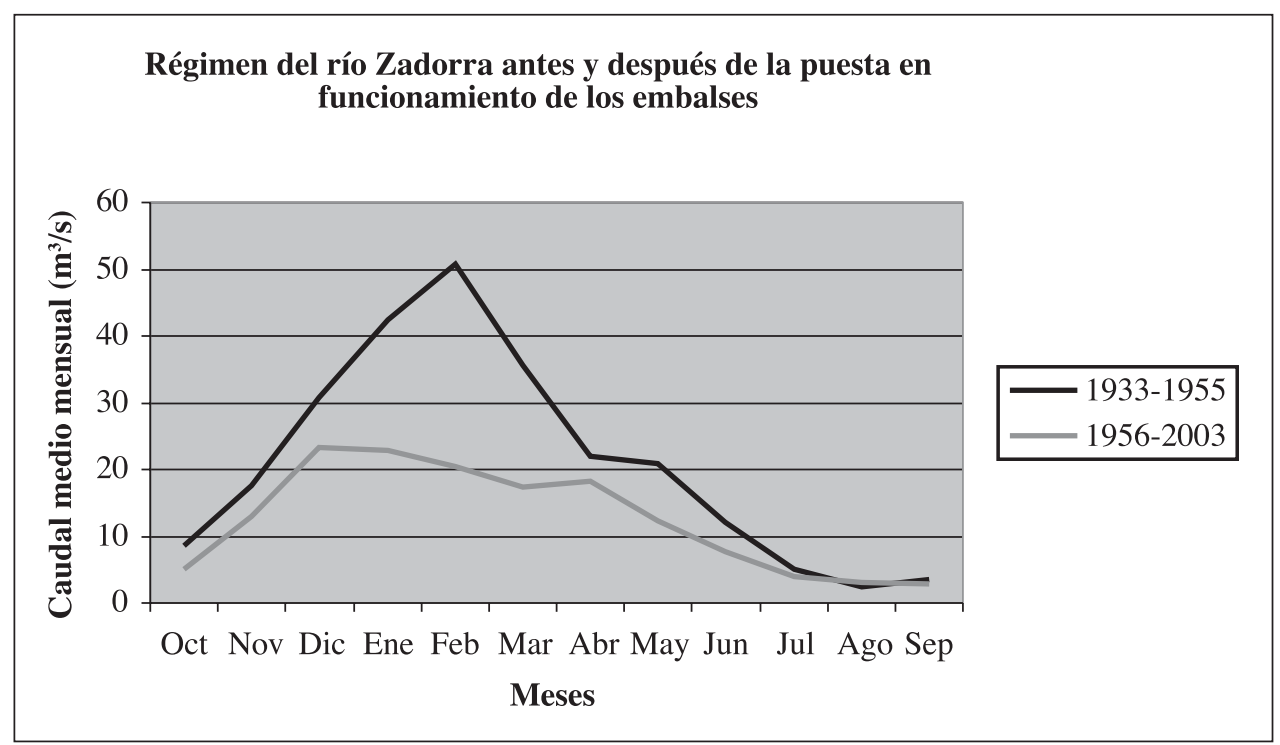

FIGURA 3. Régimen del río Zadorra antes y después del inicio en la explotación de los embalses. (Fuente: CHE. Elaboración propia).

En lo que a los eventos de crecida se refiere, tal y como señala la Figura 4, se ha producido una disminución en la frecuencia de los mismos a raíz del comienzo en la explotación de los embalses, debido a la laminación ejercida por éstos, pasando de frecuencias de 6 crecidas por año, a una media de una por año. Esta disminución en un principio era menos patente, debido a que la gestión de los embalses no estaba todavía ajustada, lo cual permitió que todavía se registrasen un número considerable de crecidas anuales.

La menor frecuencia en las crecidas ha supuesto en general una disminución de la peligrosidad de las mismas, sin embargo este hecho ha propiciado la falsa sensación de seguridad que ha llevado a la invasión de determinados terrenos, lo cual ha incrementado la exposición. A ello se añade que la menor frecuencia de crecidas ordinarias y la disminución de los caudales en general, ha generado una fijación de finos y la invasión del cauce por parte de la vegetación, que ha disminuido la capacidad de desagüe, incrementando la posibili- 


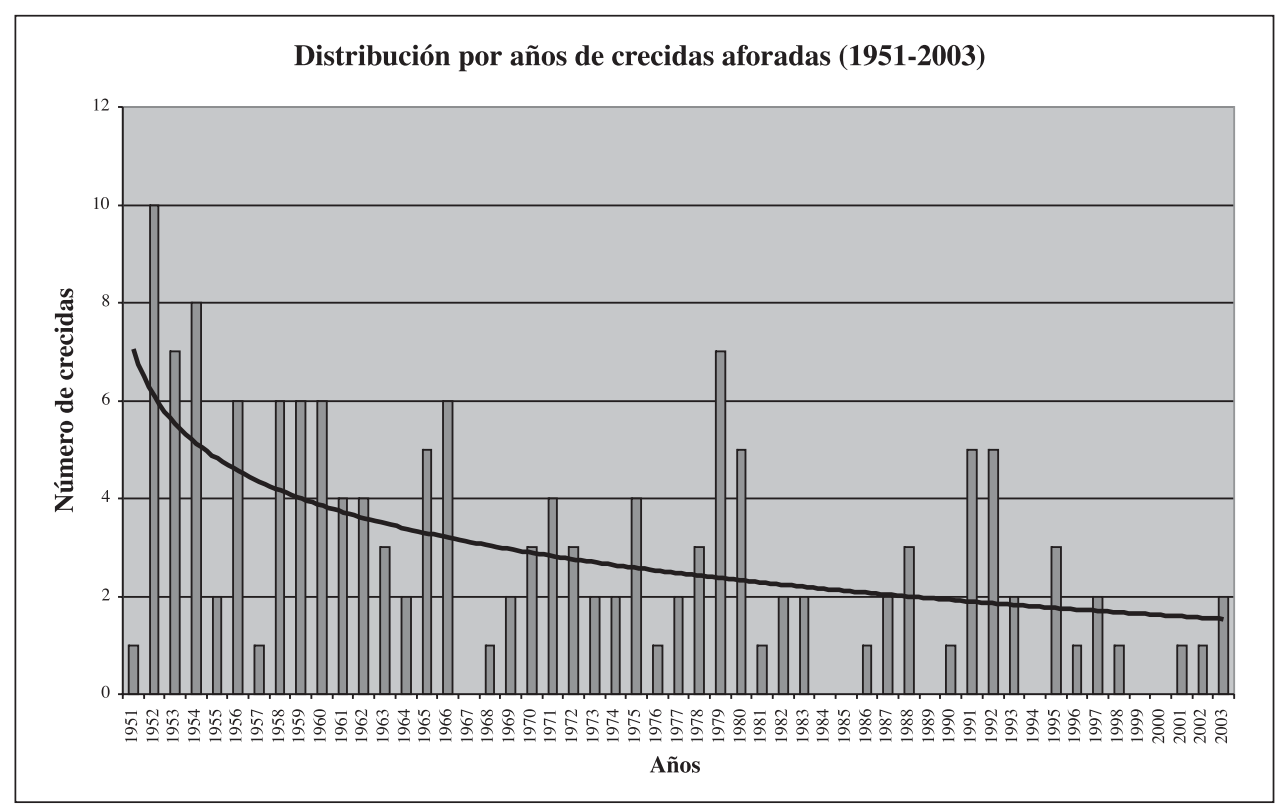

FIGURA 4. Distribución de crecidas por años (1951-2003). Elaboración propia.

dad de desbordamientos con caudales menores, ello supone que crecidas que en principio son de menor entidad, por una menor caudalosidad, terminan presentando mayores desbordamientos.

\section{La peligrosidad de las crecidas del Zadorra}

La peligrosidad señala la intensidad del proceso o suceso natural, y su valoración es independiente de su afección a los seres humanos o sus actividades (PANIZZA, 1988). El peligro de crecidas depende únicamente del funcionamiento hidrológico e hidráulico de la cuenca y de su red hidrográfica y puede analizarse con métodos hidrológicos e hidráulicos (GILARD, 1997). Autores como DUNNE y LEOPOLD (1978) establecieron algunos de los elementos de peligrosidad de las crecidas. En el caso de las crecidas del Zadorra su peligrosidad viene dada por una serie de factores, como son la magnitud o intensidad, la extensión espacial del peligro asociado a las crecidas y los lugares afectados, la periodicidad o frecuencia de las mismas, y finalmente la gestión de los embalses.

El tipo de crecidas que predominan en el río Zadorra, son de valle, más o menos lentas y previsibles. De hecho en el curso principal del Zadorra sólo se ha conocido un único evento de crecida relámpago, registrado en julio de 1988 en el tramo alto del río.

En cuanto a la magnitud de las crecidas, expresado mediante su caudal, se puede señalar que en la serie de datos que va desde 1951 a 2003, se han registrado crecidas con tan solo $78,10 \mathrm{~m}^{3} / \mathrm{s}$ de caudal máximo instantáneo, registrado el 2 de abril de 1975, cerca del umbral de 5 veces el caudal medio de la serie, establecido como umbral de crecida. El mayor caudal máximo instantáneo registrado ha sido aproximadamente de $600,72 \mathrm{~m}^{3} / \mathrm{s}$ (ya que el propio desbordamiento del río en el entorno de la estación de aforo impidió su exacta medición), obtenido el 5 de febrero de 2003 en la estación de aforo de Arce, cerca de la 
desembocadura del Zadorra en el río Ebro, que en términos de caudal específico rondaba los $441,29 \mathrm{l} / \mathrm{s} / \mathrm{km}^{2}$. Sin embargo probablemente en otros eventos pudieran haberse registrado caudales superiores en determinados lugares, que vieron su registro reducido por la propia laminación natural de la crecida. Del total de crecidas recogidas desde que existen registros foronómicos de manera continuada, consideramos que 7 tienen carácter de extraordinarias (Tabla 1).

Tabla 1.

CRECIDAS EXTRAORDINARIAS (1951-2003)

\begin{tabular}{|c|c|}
\hline Fecha & Q máx. inst. $\left(\mathbf{m}^{\mathbf{3} / \mathbf{s})}\right.$ \\
\hline Diciembre 1959 & 430 \\
\hline Noviembre 1961 & 467 \\
\hline Junio 1977 & 513,5 \\
\hline Diciembre 1980 & 539,54 \\
\hline Enero 1981 & 586,04 \\
\hline Diciembre 1993 & 550 \\
\hline Febrero 2003 & 600,72 \\
\hline
\end{tabular}

Fuente: Confederación Hidrográfica del Ebro. Elaboración propia.

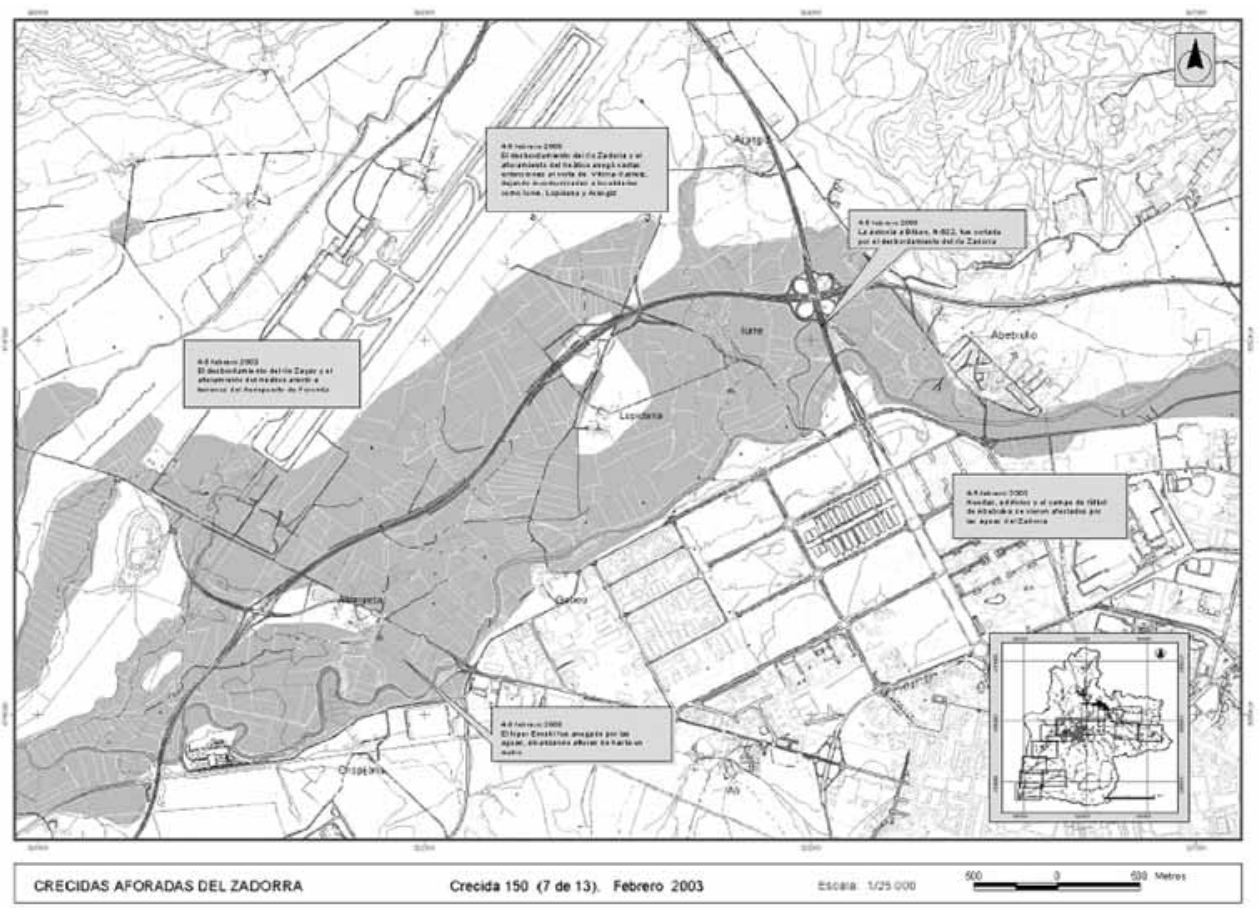

FIGURA 5. Crecida de febrero de 2003 en el entorno de la ciudad de Vitoria-Gasteiz. (Fuente: Trabajo de campo. Elaboración propia). 
El estudio de la extensión espacial del peligro y el análisis de los lugares más afectados, se ha realizado mediante la representación cartográfica de todas las crecidas con desbordamiento registradas desde 1952, a partir de la información obtenida en expedientes administrativos, prensa, crónicas y trabajo de campo. Las mayores extensiones de la lámina de agua desbordada se producen en las inmediaciones de la ciudad de Vitoria-Gasteiz (Figura 5), de un lado por el propio desbordamiento del río y de otro por el afloramiento de las aguas del acuífero cuaternario sobre el que se asienta la ciudad. Sin embargo, las superficies anegadas no adquieren grandes profundidades ni fuertes velocidades del agua, por situarse sobre terrenos muy llanos, con escasas pendientes, donde las aguas se expanden fácilmente en la horizontal.

El análisis de la periodicidad para hallar los periodos de retorno se ha realizado a través de la distribución de GUMBEL $(1934,1945,1958)$ con la serie de datos que va de 1952 a 2003, donde se ha conseguido un ajuste adecuado (Figura 6). El rango de periodos de retorno es muy amplio, oscilando entre un escaso año y los 44 años de la crecida con mayor caudal instantáneo registrado, la de febrero de 2003.

De un análisis más pormenorizado se denota la influencia que los embalses han tenido en el comportamiento hidrológico extremo de las crecidas. En la Figura 7 se aprecia la disminución de los periodos de retorno de las crecidas ordinarias, es decir, la proliferación de crecidas de menor magnitud y que producen desbordamientos. Lo cual vuelve a remarcar la menor capacidad del cauce para desaguar el agua, por la fijación de finos y la invasión del

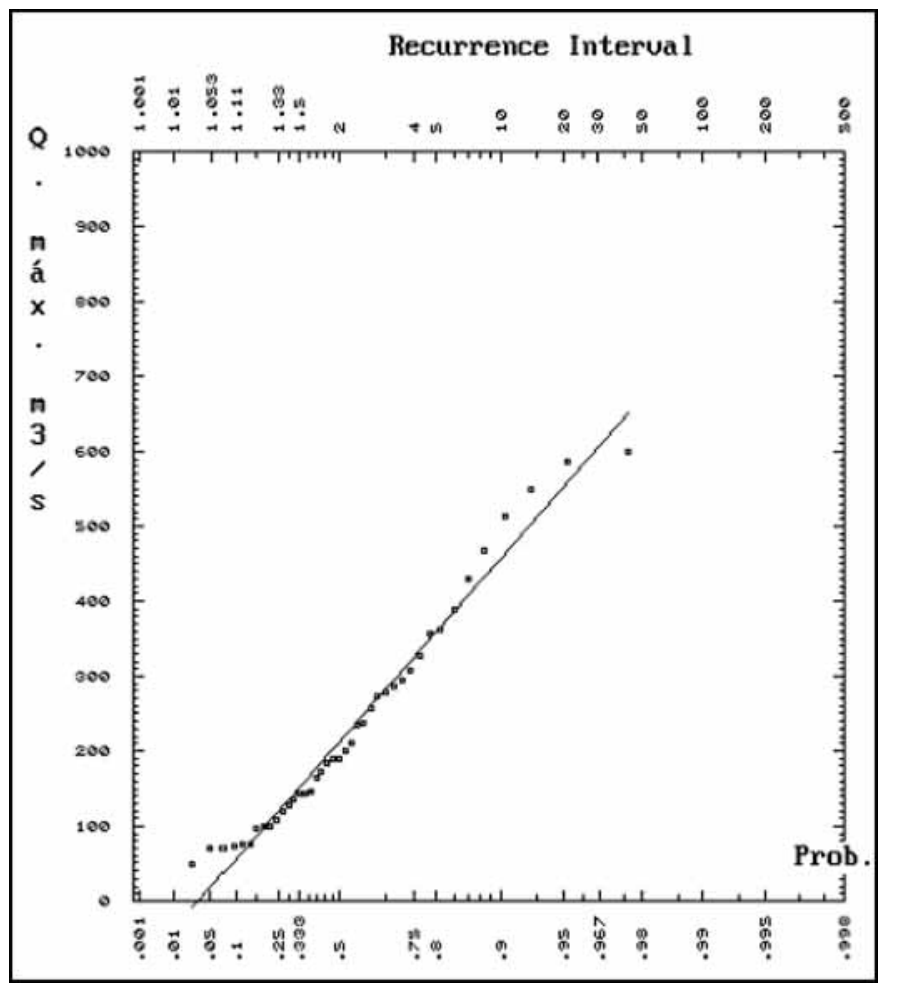

FiguRA 6. Ajuste de Gumbel. (Fuente: RANKPLOT. Elaboración propia). 


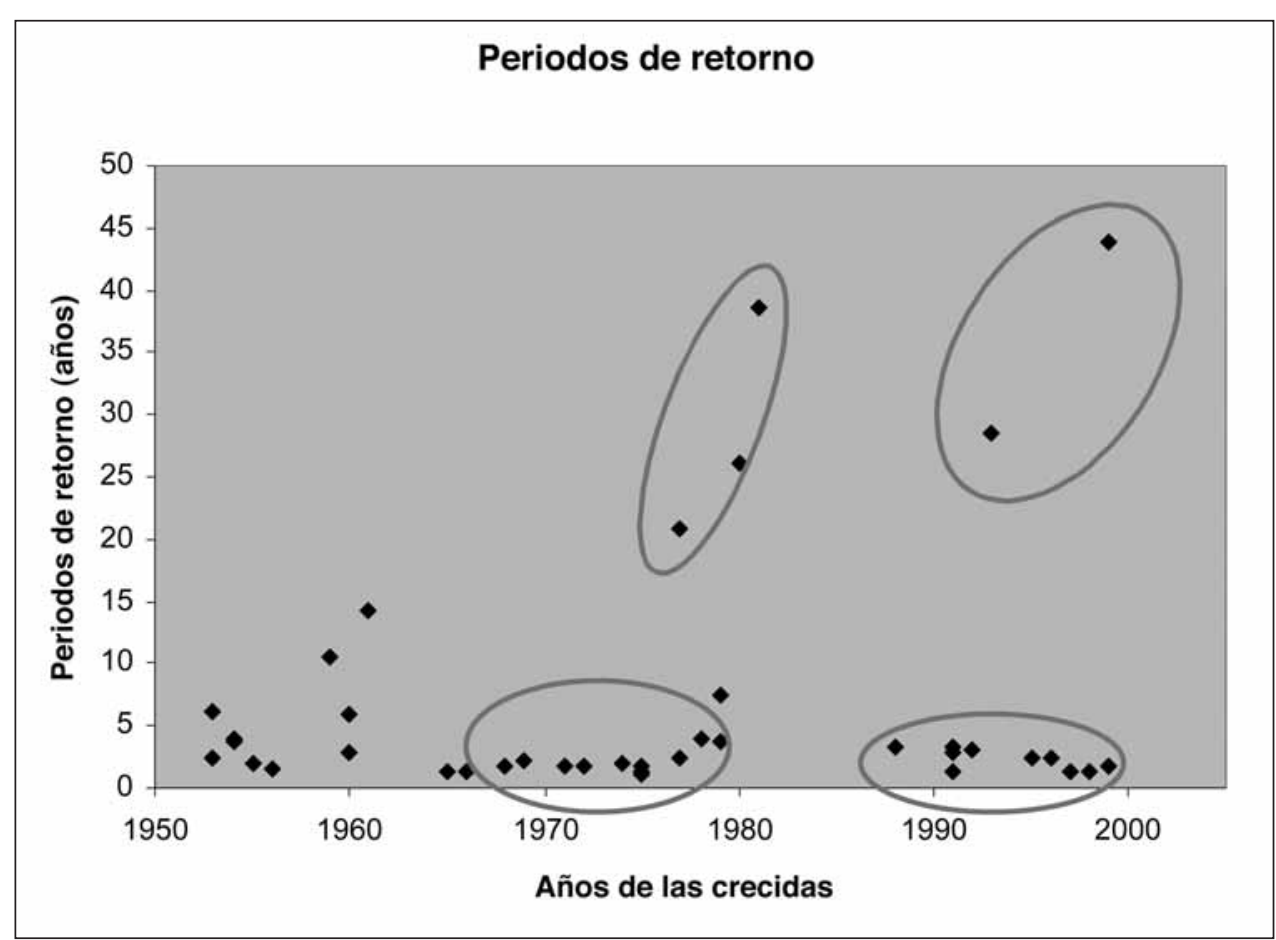

FIGURA 7. Periodos de retorno de las crecidas del Zadorra. (Fuente: Confederación Hidrográfica del Ebro. Elaboración propia).

mismo por parte de la vegetación. Por otro lado también se aprecia la inviabilidad de los embalses en la reducción de aquéllas con periodos de retorno grandes, y que por tanto presentan caudales altos, que siguen produciéndose.

El último elemento de la peligrosidad de las crecidas se manifiesta en la gestión de los embalses, que se ha convertido en uno de los más determinantes. Los diferentes usos a los que están destinados, que resultan de intereses contradictorios, complica la gestión de los mismos, lo cual lleva a un ajuste de las curvas de explotación-garantía y seguridad, poniendo al límite la situación en momentos de avenida. A ello está unido la coincidencia en la confluencia de las aguas altas de determinados afluentes, lo cual incrementa la onda de avenida. Por tanto estas infraestructuras demuestran su incapacidad en hacer desaparecer las crecidas más dañinas, es más, en algún evento han supuesto un factor de incremento de la peligrosidad, al haberse ajustado al máximo las curvas de seguridad y garantía, y haberse retrasado los desembalses, obligando a realizarlos posteriormente de forma repentina.

\section{Los espacios inundables, la vulnerabilidad del territorio y las actividades}

El análisis de la vulnerabilidad es el segundo aspecto a analizar en un estudio de riesgos. Estudio que se aborda por un lado con la identificación de los espacios inundables, y los usos establecidos sobre los mismos. Estos espacios quedan bien señalados en la representación de la llanura de inundación que se ha elaborado mediante el método hidrogeomorfológico. 


\subsection{La representación de la llanura de inundación}

El método hidrogeomorfológico tiene por objeto identificar los elementos de referencia que permiten interpretar la dinámica de los espacios fluviales, zonas activas de evolución de ríos y riberas, y las unidades geomorfológicas que constituyen los diferentes lechos (MASSON et al, 1996). Para la delimitación de estos espacios se ha empleado la fotografía aérea de 1968 y 1998, a escala 1:20.000 y 1:22.000 respectivamente. Los elementos indicadores de esa llanura de inundación son diversos: taludes, bordes de terrazas, tipo de vegetación, localización de construcciones, edificaciones antiguas, etc. (ROSSELLÓ, 1989; MASSON, 1996). Posteriormente hay que realizar la verificación de la representación obtenida mediante el trabajo de campo, para aclarar incertidumbres y estudiar los elementos no aparentes sobre las fotografías (como límites u obras de defensa que puedan estar enmascaradas por una densa vegetación). Todos estos elementos han servido de identificadores de la llanura aluvial. Hay que tener en cuenta, sin embargo, que a pesar de que «las sociedades humanas han buscado emplazamientos que aminoraran el peligro, la acreción vertical y horizontal del llano de inundación es un factor dinámico que juega en contra de espacios que pudieran estar libres de inundación en los momentos fundacionales de las ciudades» (MATEU, 1990) y que posteriormente pudieran verse afectados por un riesgo que antes no presentaban.

Sin embargo a la hora de delimitar la llanura de inundación hay que tener en cuenta, que podemos encontrar morfologías heredadas del pasado, siendo necesaria la valoración de su funcionalidad actual, y de otro lado las actuaciones antrópicas realizadas en el cauce y la llanura que hayan podido modificar la capacidad del lecho menor, a través de dragados, canalizaciones, ensanchamientos etc., que transforman radicalmente las condiciones de desbordamiento. Estas obras suelen afectar más a las ríos de menor entidad, lo cual incide en el efecto relámpago de las crecidas en las llanuras de inundación de la cuenca alta y media, en detrimento de la seguridad aguas abajo. Este sería el caso de la única crecida relámpago de la que se tiene constancia, registrada el 19 de julio de 1988 en el tramo alto del río. Con origen en una fuerte tormenta, las aguas del río crecieron, pero las obras de canalización y defensa realizadas aguas arriba, así como la rectificación y profundización acometida, bien con motivo de la concentración parcelaria, como con fines de defensa contra inundaciones, aceleraron y trasladaron el conjunto de aguas crecidas aguas abajo, afectando a una vivienda situada sobre terrenos inundables de manera repentina.

A escala de la llanura aluvial moderna el método hidrogeomorfológico presenta resultados muy interesantes constatados en diversos cursos fluviales franceses (MASSON, 1996). Finalmente, para validar la representación cartográfica obtenida se empleó la crecida de febrero de 2003, ya que tal y como señalaron WILLIAMS y COSTA (1988), la cartografía más fidedigna se obtiene después de una inundación. La comprobación llevó a una validación bastante general de la mancha de inundación obtenida, puesto que coincidía en bastantes puntos, sin embargo sí que se produjeron desbordamientos en lugares que no se habían señalado, que pudieran haberse visto afectadas por los efectos que tuvo la canalización del río Alegría, afluente que le llega al río por la margen izquierda antes de rodear la ciudad de Vitoria-Gasteiz por el norte, que disminuyó la laminación natural y trasladó las aguas crecidas hacia aguas abajo (Figura 8).

La llanura de inundación obtenida presenta una superficie aproximada de $23,9 \mathrm{~km}^{2}$ a lo largo de todo el cauce del río Zadorra y sobre ese espacio se sitúan terrenos de cultivo, viviendas, infraestructuras e industrias. La mayor parte de la superficie está ocupada por la primera de las actividades, sin embargo los daños más graves y cuantiosos se producen en viviendas, infraestructuras e industrias. 


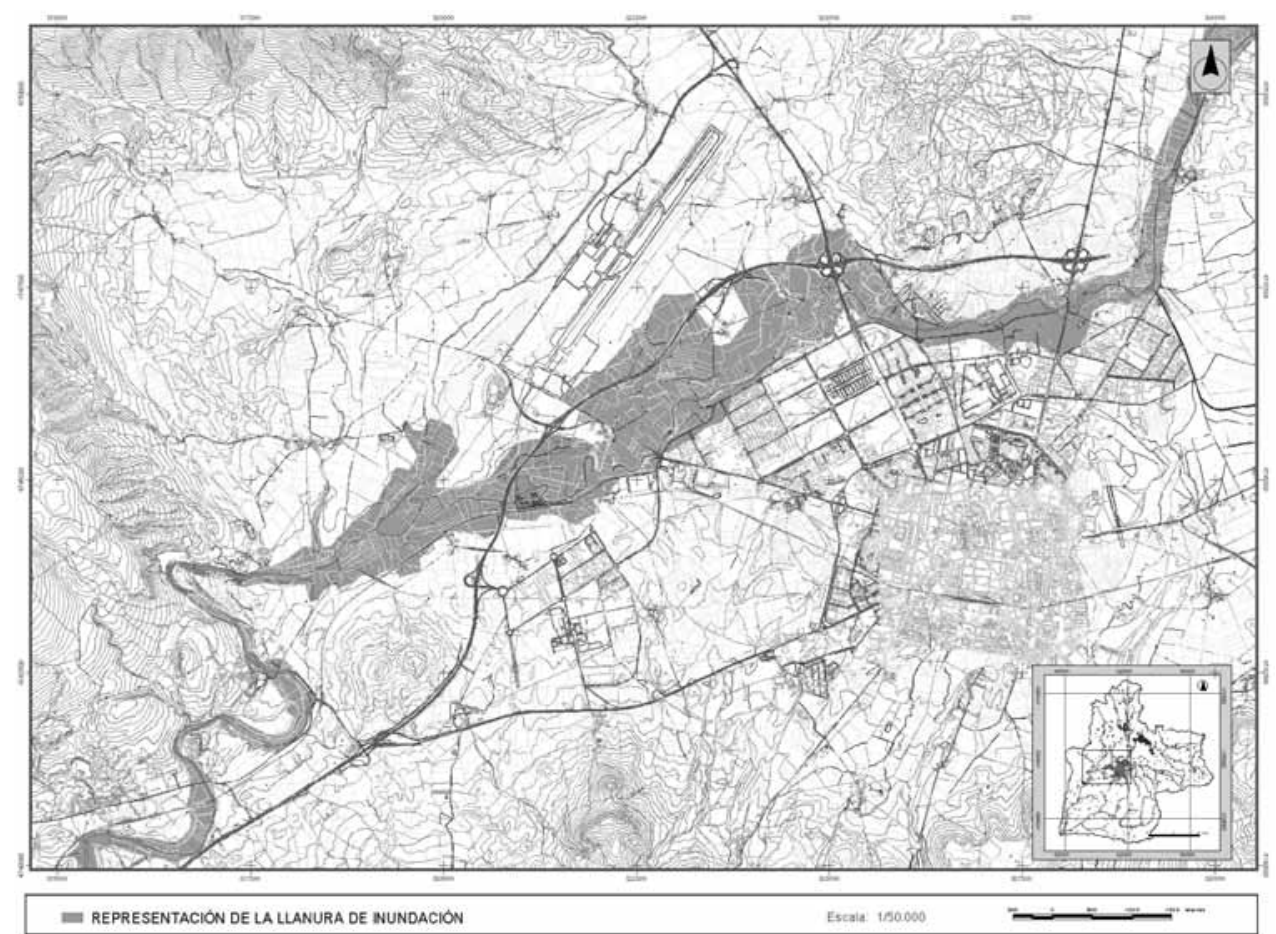

FIGURA 8. Representación de la llanura de inundación en el sector más próximo a Vitoria-Gasteiz. (Fuente: Trabajo de campo. Elaboración propia).

\subsection{Variación de la vulnerabilidad del territorio}

Tal y como ya se ha señalado en el análisis de la peligrosidad, para obtener un buen conocimiento del funcionamiento de las crecidas del río Zadorra, se han realizado las representaciones cartográficas de todas las crecidas que han presentado desbordamiento desde 1952, de manera que se han podido conocer los lugares más afectados por estos procesos y la variación que han presentado a lo largo del tiempo.

De este análisis se ha constatado una modificación de los espacios potencialmente inundables y más afectados por las crecidas del río Zadorra. Durante el periodo previo a la construcción de los embalses los lugares más afectados eran aquéllos correspondientes al tramo alto del río Zadorra (Figura 9), terrenos hoy en día situados aguas arriba del embalse de Ullibarri-Ganboa. Sin embargo la actuación sobre el río con medidas como la profundización y rectificación (Figura 10), ha sobredimensionado y artificializado el cauce, de manera que ya no se ven tan afectados, aunque tal y como se pudo constatar en la crecida de febrero de 2003 siguen anegándose.

Posteriormente la puesta en explotación de los embalses ha incrementado la afección de los terrenos situados aguas abajo, debido a los factores ya comentados previamente, los repentinos desembalses, una menor capacidad de desagüe y la invasión de la llanura y cauce para uso agrícola, industrial y residencial. De manera que si bien anteriormente también 


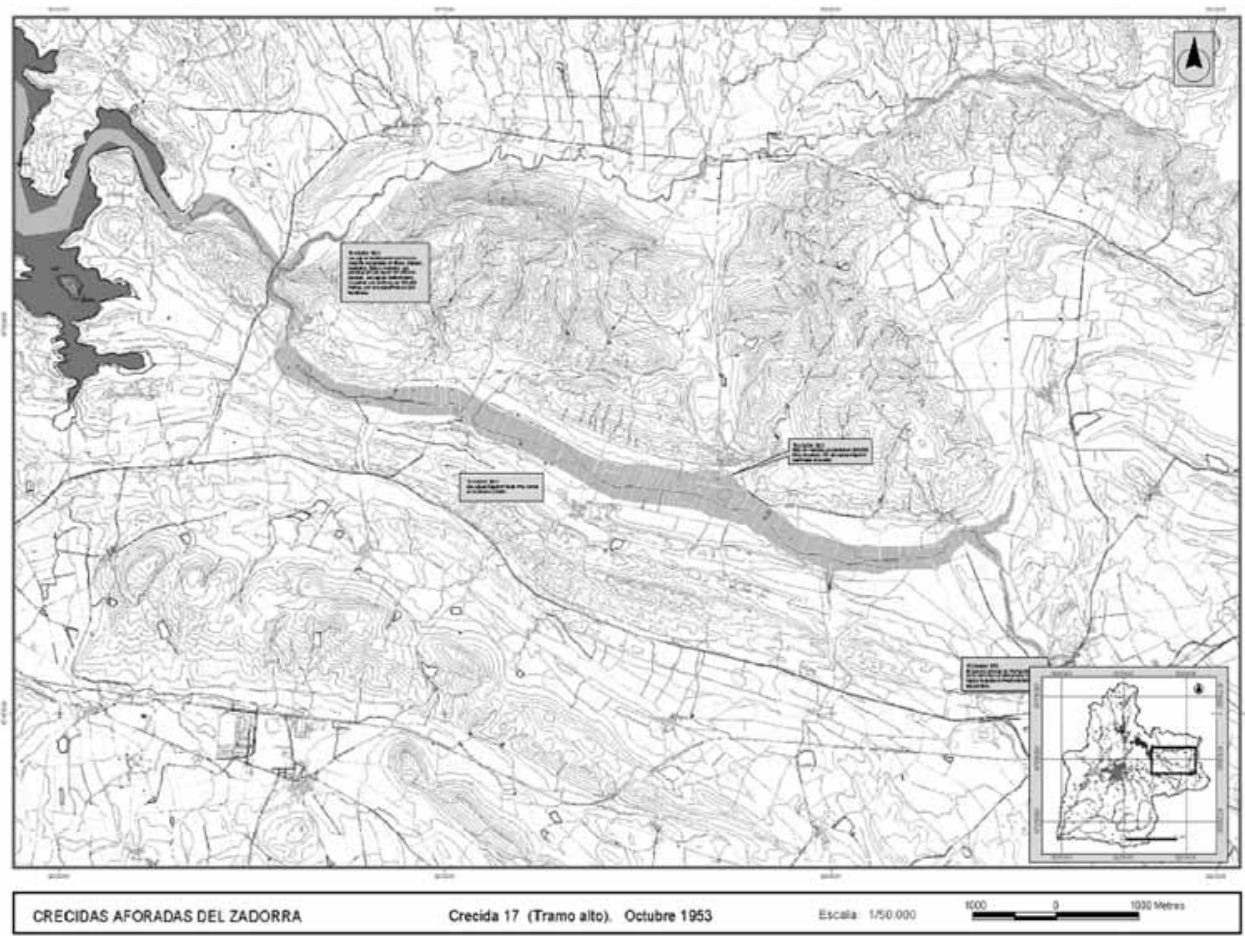

FigurA 9. Tramo alto del Zadorra en la crecida de Octubre de 1953. (Fuente: Trabajo de campo. Documentación. Elaboración propia).

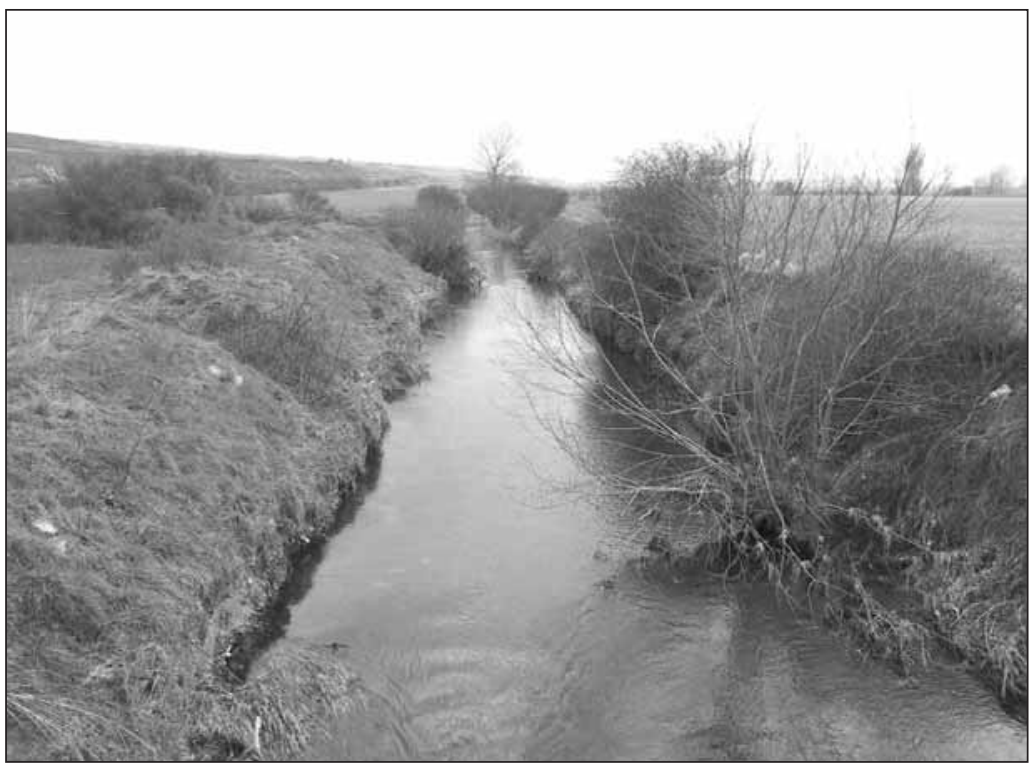

FIGURA 10. Cauce del río Zadorra en su tramo alto, rectificado y profundizado. 
estos terrenos se veían afectados, posteriormente su inundabilidad se vio notablemente incrementada. La peligrosidad aumentó por los fuertes desembalses y la coincidencia con los caudales punta de los afluentes, debido también en parte a las obras realizadas en los tramos altos de algunos de ellos que motivaron una disminución de los tiempos de concentración y una mayor aportación de caudales hacia aguas abajo. Algunas de esas obras se realizaron con motivo de la concentración parcelaria, que rectificó y profundizó los cauces y eliminó la vegetación de ribera, lo cual transportaba las aguas con mayor rapidez y eliminaba la capacidad de freno y laminación natural de los propios ríos.

Sin embargo la mayor vulnerabilidad viene dada por la progresiva invasión realizada de estos espacios inundables. El inicio de la explotación de los embalses coincidió con la industrialización de la ciudad de Vitoria-Gasteiz a mediados de los años 50, situándose los nuevos polígonos industriales sobre una zona de recurrente expansión de las aguas, al norte de la ciudad, en la confluencia de los ríos Alegría y Santo Tomás en el curso principal del Zadorra. Estos lugares históricamente inundables no habían sido ocupados, y sufrían inundaciones periódicas, que se aceptaban por parte de los que entonces cultivaban esas tierras. Pero la puesta en explotación de los embalses generó una falsa sensación de seguridad que motivó su ocupación. La vulnerabilidad de la zona queda remarcada cuando por parte de la Comisaría de Aguas del Ebro en el año 1966 considera «la zona industrial de Vitoria y caseríos colindantes» como punto de mayor riesgo, señalando como causa la «explotación inadecuada de los embalses de Ullibarri-Ganboa y Urrunaga por Aguas y Saltos del Zadorra» (Expediente municipal, 54/071/004).

Por otro lado, los escasos caudales circulantes liberados del pantano a través del caudal ecológico y la eliminación de las crecidas ordinarias, habituales anteriormente, han provocado la invasión del cauce por vegetación y maleza y la deposición de lodos que el agua es incapaz de arrastrar, lo que impide un desagüe adecuado de las aguas. De ahí que se haya indicado que, un desembalse de escasos $30 \mathrm{~m}^{3} / \mathrm{s}$, es capaz de provocar inundaciones, tal y como ya se señalaba en diciembre 1996. Responsables de Iberdrola indicaron entonces que con «el desembalse de $30 \mathrm{~m}^{3} / \mathrm{s}$ ya empiezan a producirse problemas, entre 30 y $60 \mathrm{~m}^{3} / \mathrm{s}$ el agua cubre algunos puentes, y con más de $60 \mathrm{~m}^{3} / \mathrm{s}$ se empieza a inundar la zona industrial de Vitoria-Gasteiz». Esto empezó a suceder bien pronto, puesto que en los primeros años de explotación, se comprobó en varias ocasiones, que era a partir de un vertido de $60 \mathrm{~m}^{3} / \mathrm{s} \mathrm{de}$ cada uno de los embalses, Ullibarri y Urrunaga, cuando se producían los desbordamientos. Anteriormente, en 1956, año de inicio de la explotación de los embalses, se llegaron a verter $122 \mathrm{~m}^{3} / \mathrm{s}$ en Ullibarri y $103 \mathrm{~m}^{3} / \mathrm{s}$ en Urrunaga, y entonces no se produjeron protestas por parte del Ayuntamiento de Vitoria por afección a los incipientes terrenos industriales y sin embargo sí que las hubo por parte de agricultores afectados, sobre todo los situados aguas abajo de la presa de Urrunaga. Ya en 1965, los caudales admisibles se redujeron a 40 y 50 $\mathrm{m}^{3} / \mathrm{s}$, según el propio Ayuntamiento de Vitoria.

Actualmente prosigue la actuación sobre el cauce del río Zadorra con medidas estructurales, especialmente en el entorno de la ciudad de Vitoria-Gasteiz, lo cual puede incidir en la disminución de los tiempos de concentración incrementando el riesgo en lugares situados aguas abajo a los de la actuación.

\section{Valoración del riesgo y conclusiones}

La valoración del riesgo demuestra que éste se ha visto incrementado, en gran medida por el aumento de la vulnerabilidad, concretado en una mayor presión urbanística sobre la llanura de inundación, pero también el de la peligrosidad, debido a la gestión de desembalses realizada. Es un riesgo que afecta fundamentalmente a las actividades económicas y a 
los usos residenciales, equipamientos e infraestructuras, siendo bastante leve el riesgo asociado a personas, dado que es muy previsible y la evacuación de la población no resulta difícil. Los puntos de mayor riesgo se sitúan en zonas urbanizadas y cercanas a localidades, como es el norte de la ciudad de Vitoria-Gasteiz y puntos donde se ha edificado en la llanura de inundación, especialmente en el tramo medio y bajo del río.

Establecer con precisión el nivel de riesgo en el lecho mayor resulta muy difícil, en principio se considera como sometido al riesgo hidrológico, y susceptible de ser afectado por crecidas de débil frecuencia (MASSON, 1996). Ese hecho debería de llevar, por precaución, a renunciar a la urbanización de ese espacio, dependiendo de las posibilidades de reubicación espacial. Más si cabe teniendo en cuenta que no es un territorio que carezca de espacio, de manera que debieran de liberarse los terrenos inundables de edificaciones.

El análisis de la peligrosidad de las crecidas en función de sus caudales y los periodos de retorno, debe de realizarse con suma cautela, dado que las cortas series de datos reducen notablemente la fiabilidad de los resultados obtenidos con las funciones estadísticas.

Se aprecia una variación de los lugares más vulnerables, lo cual ha incidido en la modificación del reflejo espacial del riesgo, trasladándose especialmente al tramo medio del río y entorno de la ciudad de Vitoria-Gasteiz. El hecho de que en muchos lugares los desbordamientos se deban al afloramiento del freático, señala la poca efectividad que algunas obras de defensa puedan presentar ante estos procesos.

Ello hace necesario una actualización del mapa de inundabilidad cada cierto tiempo, para actualizar los cambios realizados en la cuenca, y las modificaciones en el funcionamiento hidrológico y de procesos de crecidas, así como las zonas de expansión de las aguas.

En definitiva la peligrosidad de las crecidas del Zadorra no es muy importante, sin embargo ésta se ha visto incrementada en los últimos años, fundamentalmente por la gestión de caudales realizada desde los embalses, lo cual hace necesario incidir en una gestión más racional de los mismos, así como un respeto escrupuloso de los espacios inundables.

\section{Agradecimientos}

A Ana Sáenz de Olazagoitia, Alfredo Ollero y Orbange Ormaetxea.

\section{Bibliografía}

AYALA, F.J.; DURÁN, J.J. y PEINADO, T. (1987): I Curso de Riesgos geológicos. Instituto Geológico y Minero de España, Madrid, 333 pp.

AYALA, F.J. y OLCINA, J. (coord.) (2002): Riesgos naturales. Ariel, Barcelona, 1.512 pp. BAKER, V.R.; KOCHEL, C.R. y PATTON, P.C. (1988): Flood Geomorphology. Wiley, New York, 503 pp.

BESCÓS, A. y CAMARASA, A.M. (1998): «Cartografía de riesgos de inundación mediante sistemas de información geográfica. Una aplicación al llano de inundación del río Arga (Navarra)», En Gómez Ortiz, A. y Salvador, F. (Eds.): Investigaciones recientes de la Geomorfología española, Sociedad Española de Geomorfología, Barcelona, pp. 703706.

BESCÓS, A. y CAMARASA, A.M. (2000): «Elaboración de cartografía de zonas inundables. Aplicación al llano de inundación del río Arga (Navarra)», en Serie Geográfica n ${ }^{\circ}$ 9, pp. 219-236.

BEVEN, K.J. y CARLING, P. (eds.) (1989): Floods: their hydrological, sedimentological and geomorphological implications. Wiley, Chichester, 304 pp. 
CALVO, F. (1984): «La Geografía de los riesgos», Geo-Crítica, nº 54, 39 pp.

CALVO, F. (1986): La ordenación del espacio ante los riesgos naturales. Actas, discursos, ponencias y nuevas mesas redondas del IX Coloquio de la Asociación de Geógrafos Españoles. Departamento de Geografía, Murcia, pp. 141-159.

CALVO, F. (1997): «Algunas consideraciones sobre geografía de los riesgos», en Scripta Nova, $\mathrm{n}^{\circ} 10,11 \mathrm{pp}$.

CALVO, F. (2001): Sociedades y territorio en riesgo. Ed. Serbal, 186 pp., Barcelona.

CAMARASA, A. y MATEU, J. (coords.) (2000): Las inundaciones en España en los últimos veinte años. Una perspectiva geográfica. Serie Geográfica, 9. Universidad de Alcalá, $253 \mathrm{pp}$

CAPEL MOLINA, J.J. (1983): Las inundaciones de agosto de 1983 en el País Vasco, Cantabria y Navarra Atlántica. La Crónica, Almería, 145 pp.

DUNNE, T. y LEOPOLD, L.B. (1978): Water in environmental planning. Freeman, San Francisco, 818 pp.

Expediente municipal 54/071/004 «Inundaciones del Zadorra», Archivo Municipal de Vitoria-Gasteiz.

FERRER, J. (2000): Análisis estadístico de caudales de avenida. CEDEX, 42 pp., Madrid.

FRANCÉS, F. (1997): Delimitación del riesgo de inundación a escala regional en la Comunidad Valenciana. Conselleria d'Obres Publiques, Urbanisme i Transports, Valencia, 72 pp +1 plan pleg.

GARCÍA-RUIZ, J.M.; PUIGDEFÁBREGAS, J. y MARTÍN, M.C. (1983): «Diferencias espaciales en la respuesta hidrológica a las precipitaciones torrenciales de noviembre de 1982 en el Pirineo central», en Estudios geográficos no XLIV (170-171): pp. 291-310.

GARCÍA-RUIZ, J.M.; WHITE, S.M.; MARTÍ, C.; VALERO, B.; ERREA, M.P. y GÓMEZ, A. (1996): La catástrofe del barranco de Arás (Biescas, Pirineo aragonés) y su contexto espacio-temporal. Instituto Pirenaico de Ecología. Campues de Aula Dei, Consejo Superior de Investigaciones Científicas, Zaragoza, $54 \mathrm{pp}$.

GIL OLCINA, A. y MORALES GIL, A. (coord.) (1989): Avenidas fluviales e inundaciones en la cuenca del Mediterráneo. Instituto Universitario de Geografía de la Universidad de Alicante. Caja de Ahorros del Mediterráneo, Universidad de Alicante, Alicante, $586 \mathrm{pp}$.

GILARD, O. (1997): «An hydrological definition of flood vulnerability»en FRIEND Flow Regimes from International Experimental and Network Data. Third report 1994-1997, pp. 381-385.

GILARD, O.; GIVONE, P.; PAQUIER, A.; GENDREAU, N.; FAURE, J.B. y HEROUN, E. (1998): «Guide practique de la méthode luondabilité», Etude interagences, $\mathrm{n}^{\circ} 60$, 158 pp., Paris.

GUMBEL, E.J. (1934). Les valeurs extrêmes des distributions statistiques. Annales de l'I.H. Poincaré, 308 pp.

GUMBEL, E.J. (1945): «Floods estimated by probability method» en Engineering News Record $\mathrm{n}^{\circ} 134$, pp. 833-837.

GUMBEL, E.J. (1958): Statistics of extremes. Columbia University Press, New York, 371 pp.

HERAS, F. (1978): Métodos prácticos de estimación de máximas crecidas. Centro de Estudios Hidrográficos, 2 vols., Madrid.

IBISATE, A. (2004): Las crecidas fluviales del Zadorra: procesos, riesgos y propuestas de ordenación. Tesis doctoral, $630 \mathrm{pp}+$ anexo cartográfico (inédito).

KUNDZEWICZ, Z.W.; ROSBJERG, D.; SIMONOVIC, S.P. y TAKEUCHI, K. (eds.) (1993): Extreme hydrological events: precipitation, floods and droughts. IAHS publ. 213, Wallingford , $460 \mathrm{pp}$. 
LAÍN, L. (ed.) (1999): Sistemas de Información Geográfica en riesgos naturales y medio ambiente. ITGE, Madrid, $277 \mathrm{pp}$.

MARTÍNEZ GIL, J. (2003): «Hidrología», en ARROJO, P. et al: Directrices para un Plan Estratégico de Gestión de Aguas en Álava. Fundación Nueva Cultura del Agua, Zaragoza, pp. 18-64.

MASSON, M.; GARRY, G. y BALLAIS, J.L. (1996): Cartographie des zones inondables: approche hydrogéomorfologique. Ministère de l'Equipement et Ministère de l'Environnement, Paris, 100 p. + annexes.

MATEU, J. (1990): «Avenidas y riesgo de inundación en los sistemas fluviales mediterráneos de la Península Ibérica», en Boletín de la Asociación de Geógrafos Españoles $\mathrm{n}^{\circ}$ 10, pp. 45-86.

MATEU, J. (1992): «La Geografía de los riesgos en España. La Geografía en España (19701990)», en Aportación Española al XXVII Congreso de la Unión Geográfica Internacional. Washington, RSG-AGE, Fundación BBV, Madrid, pp. 241-245.

OLCINA, J. (1994): Riesgos climáticos en la Península Ibérica. Libros Penthalon, Madrid, $440 \mathrm{pp}$

PANIZZA, M. (1988): Geomorfologia applicata. Metodi di applicazione alla pianificazione territoriale e alla valutazione d'impatto ambientale. Nuova Italia Scientifica, Roma, $342 \mathrm{pp}$.

ROSSELLÓ. V.M. (1983): «La formación de la riada del Júcar (20/21 oct. 1982) aguas arriba de Tous», en Estudios geográficos n ${ }^{\circ}$ XLIV, 170-171, pp. 171-186.

ROSSELLÓ, V.M. (1983): «La revinguda del Xúquer i el desastre de la Ribera (20-21 octubre 1982). Una perspectiva geogràfica», en Cuadernos de Geografía no 32-33, pp. 3-38.

ROSSELLÓ, V.M. (1989): «Los llanos de inundación», en GIL OLCINA, A. y MORALES GIL, A. (coord.): Avenidas fluviales e inundaciones en la cuenca del Mediterráneo. Instituto Universitario de Geografía de la Universidad de Alicante. Caja de Ahorros del Mediterráneo, Alicante, pp. 243-283.

SAURÍ, D. y RIBAS, A. (1994): «El análisis del riesgo de avenida en las escuelas geográficas anglosajona, francesa y española», en Estudios geográficos $\mathrm{n}^{\circ} \mathrm{LV}, 216$, pp. 481502 .

WILLIAMS, G.P. y COSTA, J.E. (1988): «Geomorphic measurements after a flood», en BAKER, V.R.; KOCHEL, R.C y PATTON, P.C. (eds.): Flood geomorphology, Wiley, New York, pp. 65-77.

WHITE, G.F. et al (1958). Changes in Urban Ocupance of Floodplains in the United States. Research Paper 57. Department of Geography. University of Chicago. 